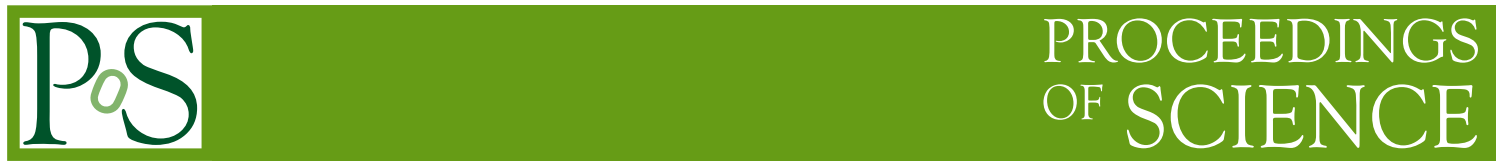

\title{
Helicity Evolution at Small $x$
}

\section{Yuri V. Kovchegov*}

Department of Physics, The Ohio State University, Columbus, OH 43210, USA

E-mail: kovchegov.1@osu.edu

\section{Daniel Pitonyak}

Division of Science, Penn State University-Berks, Reading, PA 19610, USA

E-mail: dpitonyak@quark.phy.bnl.gov

\section{Matthew D. Sievert}

Theoretical Division, Los Alamos National Laboratory, Los Alamos, NM 87545, USA

E-mail: sievertmdelanl.gov

We construct small- $x$ evolution equations which can be used to calculate quark and anti-quark helicity TMDs and PDFs, along with the $g_{1}$ structure function. These evolution equations resum powers of $\alpha_{s} \ln ^{2}(1 / x)$ in the polarization-dependent evolution along with the powers of $\alpha_{s} \ln (1 / x)$ in the unpolarized evolution which includes saturation effects. The equations are written in an operator form in terms of polarization-dependent Wilson line-like operators. While the equations do not close in general, they become closed and self-contained systems of non-linear equations in the large- $N_{c}$ and large- $N_{c} \& N_{f}$ limits.

QCD Evolution 2016

May 30-June 03, 2016

National Institute for Subatomic Physics (Nikhef), Amsterdam

\footnotetext{
${ }^{*}$ Speaker.
} 


\section{Introduction}

These proceedings are based on the work done in [1].

Our aim is to derive perturbative QCD prediction for the asymptotic small Bjorken $x$ behavior of the quark and gluon helicity distribution functions and for related observables. In these proceedings we will concentrate on the flavor-singlet quark helicity distribution $\Delta q\left(x, Q^{2}\right)$. We will derive helicity evolution equations resumming powers of $\alpha_{s} \ln ^{2}(1 / x)$ with $\alpha_{s}$ the strong coupling constant: this resummation is referred to as the double-logarithmic approximation (DLA). These evolution equations allow one to determine the leading perturbative behavior of the small- $x$ asymptotics of $\Delta q\left(x, Q^{2}\right)$ (see $\left.[2,3]\right)$. Such theoretical input is necessary to assist the efforts to determine the small- $x$ part of the quark contribution to proton spin

$$
S_{q}\left(Q^{2}\right)=\frac{1}{2} \int_{0}^{1} d x \Delta \Sigma\left(x, Q^{2}\right), \quad \Delta \Sigma\left(x, Q^{2}\right)=[\Delta u+\Delta \bar{u}+\Delta d+\Delta \bar{d}+\ldots]\left(x, Q^{2}\right),
$$

where the helicity parton distribution functions (hPDFs) are

$$
\Delta f\left(x, Q^{2}\right) \equiv f^{+}\left(x, Q^{2}\right)-f^{-}\left(x, Q^{2}\right)
$$

The ultimate goal of determining the proton spin carried by quarks [and the spin carried by the gluons, $\left.S_{G}\left(Q^{2}\right)\right]$ is to resolve the proton spin crisis.

\section{The Observables}

The small- $x$ helicity observables can be obtained by studying the cross section for semiinclusive deep inelastic scattering (SIDIS) on a longitudinally polarized target, $\gamma^{*}+\vec{p} \rightarrow \vec{q}+X$. The contributions are shown diagrammatically in Fig. 1 (see [4] for a derivation).
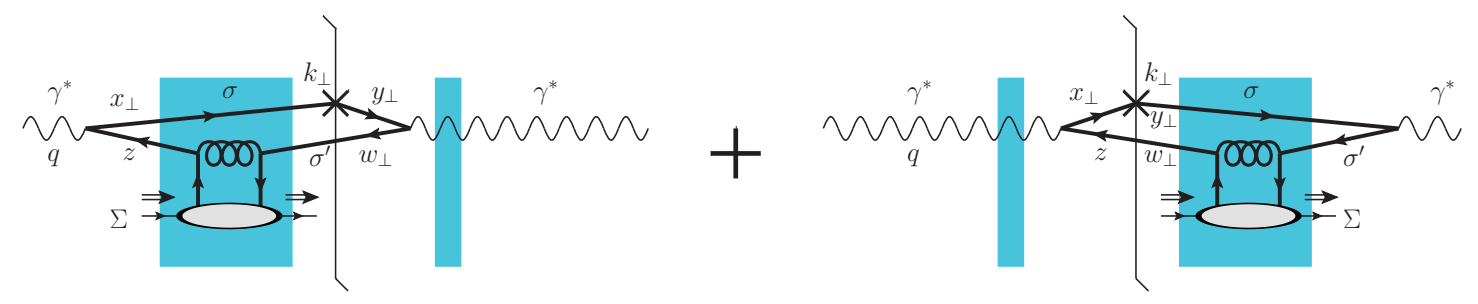

Figure 1: Diagrams contributing to the small- $x$ SIDIS process on a longitudinally polarized target, and to quark helicity TMD $g_{1 L}^{q}\left(x, k_{T}\right)$.

The corresponding quark helicity transverse momentum-dependent parton distribution function (TMD) is [1]

$$
g_{1 L}^{S}\left(x, k_{T}^{2}\right)=\frac{8 N_{c}}{(2 \pi)^{6}} \sum_{f} \int_{z_{i}}^{1} \frac{d z}{z} \int d^{2} x_{\perp} d^{2} y_{\perp} e^{-i \underline{k} \cdot(\underline{x}-\underline{y})} \frac{\underline{x}-\underline{w}}{|\underline{x}-\underline{w}|^{2}} \cdot \frac{\underline{y}-\underline{w}}{|\underline{y}-\underline{w}|^{2}} d^{2} w_{\perp} G_{\underline{x}, \underline{w}}(z) .
$$


The notation is explained in Fig. 1. Here $\underline{k}=\left(k^{x}, k^{y}\right)$ denotes transverse vectors, with $k_{\perp}=|\underline{k}|$. Variable $z$ denotes the fraction of the virtual photon's longitudinal momentum carried by the antiquark with $z_{i}=\Lambda^{2} / s$, where $\Lambda$ is the infra-red (IR) cutoff, and $s$ is the SIDIS center-of-mass energy squared. The object $G$ is the polarized dipole amplitude, which is defined by [1]

$$
\begin{aligned}
& G_{10}(z) \equiv \frac{1}{2 N_{c}}\left\langle\left\langle\operatorname{tr}\left[V_{\underline{0}} V_{\underline{1}}^{p o l \dagger}\right]+\operatorname{tr}\left[V_{\underline{1}}^{p o l} V_{\underline{0}}^{\dagger}\right]\right\rangle\right\rangle(z)=G\left(\underline{x}_{1}, \underline{x}_{0}, z\right)=G\left(\underline{x}_{10}, \underline{b}, z\right), \\
& G\left(x_{01}^{2}, z\right) \equiv \int d^{2} b G_{10}(z),
\end{aligned}
$$

where $\underline{b}=(1 / 2)\left(\underline{x}_{1}+\underline{x}_{0}\right)$. The propagator of an eikonal quark with polarization $\sigma$ in the background quark or gluon field of the target proton is written as

$$
V_{\underline{x}}(\sigma) \equiv V_{\underline{x}}+\sigma V_{\underline{x}}^{p o l}
$$

where

$$
V_{\underline{x}} \equiv \operatorname{Pexp}\left[i g \int_{-\infty}^{\infty} d x^{+} A^{-}\left(x^{+}, 0^{-}, \underline{x}\right)\right]
$$

is the light-cone Wilson line, and $V^{p o l}$ is the helicity-dependent sub-eikonal correction. The double angle brackets indicate averaging in the target wave function, with an inverse factor of center-ofmass energy squared scaled out:

$$
\langle\ldots\rangle(z)=\frac{1}{z s}\langle\langle\ldots\rangle\rangle(z) .
$$

The polarized dipole amplitude can be used to obtain other helicity observables. The flavorsinglet quark helicity PDF,

$$
\Delta q^{S}\left(x, Q^{2}\right) \equiv \sum_{f}\left[\Delta q^{f}\left(x, Q^{2}\right)+\Delta \bar{q}^{f}\left(x, Q^{2}\right)\right]
$$

at small- $x$ is equal to

$$
\Delta q^{S}\left(x, Q^{2}\right)=\frac{N_{c}}{2 \pi^{3}} \sum_{f} \int_{z_{i}}^{1} \frac{d z}{z} \int_{\frac{1}{z s}}^{\frac{1}{z Q^{2}}} \frac{d|\underline{x}-\underline{w}|^{2}}{|\underline{x}-\underline{w}|^{2}} G\left(|\underline{x}-\underline{w}|^{2}, z\right) .
$$

The $g_{1}$ structure function is

$$
\begin{aligned}
g_{1}^{S}\left(x, Q^{2}\right)=\frac{N_{c}}{2 \pi^{2} \alpha_{E M}} \sum_{f} \int_{z_{i}}^{1} \frac{d z}{z^{2}(1-z)} \int d|\underline{x}-\underline{w}|^{2} & {\left[\frac{1}{2} \sum_{\lambda \sigma \sigma^{\prime}}\left|\psi_{\lambda \sigma \sigma^{\prime}}^{T}\right|_{\left(|\underline{x}-\underline{w}|^{2}, z\right)}^{2}+\sum_{\sigma \sigma^{\prime}}\left|\psi_{\sigma \sigma^{\prime}}^{L}\right|_{\left(\underline{\mid x}-\left.\underline{w}\right|^{2}, z\right)}^{2}\right] } \\
& \times G\left(|\underline{x}-\underline{w}|^{2}, z\right)
\end{aligned}
$$

where $\psi^{T}$ and $\psi^{L}$ are the well-known light cone wave functions for the $\gamma^{*} \rightarrow q \bar{q}$ splitting (see e.g. [1]).

Our aim is to find the small- $x$ evolution equations for the polarized dipole amplitude $G_{10}(z)$. Once $G_{10}(z)$ is found, we can use Eqs. (2.1), (2.7) and (2.8) to construct the flavor-singlet quark helicity TMD, PDF and the $g_{1}$ structure function. 


\section{Large- $N_{c}$ Limit}

Similar to the case of JIMWLK evolution and Balitsky hierarchy, the general evolution equation for $G_{10}(z)$ does not close: on its right-hand side it contains operator expectation values other than $G_{10}(z)$. The operators on the right-hand side contain higher number of Wilson lines than $G_{10}(z)$. This leads to the helicity evolution analogue of the Balitsky hierarchy.

However, also similar to the unpolarized (BK) case, the evolution equations become closed equations involving $G_{10}(z)$ in the large- $N_{c}$ limit. In addition, specific to the helicity evolution case at hand, evolution equations also close in the large- $N_{c} \& N_{f}$ limit.

Here we simply quote the results, referring the reader to the derivation details in $[1,3]$. Similar to [5], our evolution equations also resum the leading-logarithmic (LLA) powers of $\alpha_{s} \ln (1 / x)$ by including the BK/JIMWLK evolved unpolarized dipole $S$-matrix

$$
\begin{aligned}
S_{01}(z) & =\frac{1}{2 N_{c}}\left\langle\left\langle\operatorname{tr}\left[V_{\underline{0}} V_{\underline{1}}^{\dagger}\right]\right\rangle\right\rangle(z)+\frac{1}{2 N_{c}}\left\langle\left\langle\operatorname{tr}\left[V_{\underline{1}} V_{\underline{0}}^{\dagger}\right]\right\rangle\right\rangle(z) \\
& \approx \frac{1}{N_{c}}\left\langle\left\langle\operatorname{tr}\left[V_{\underline{0}} V_{\underline{1}}^{\dagger}\right]\right\rangle\right\rangle(z),
\end{aligned}
$$

where we assume that

$$
\operatorname{tr}\left[V_{\underline{0}} V_{\underline{1}}^{\dagger}\right]=\operatorname{tr}\left[V_{\underline{1}} V_{\underline{0}}^{\dagger}\right]
$$

which is true at LLA. Note that LLA terms of the pure helicity evolution are not systematically included in this approach: hence we do not have a complete LLA calculation, and our results are strictly correct only in the DLA limit where $S_{01}(z)=1$.

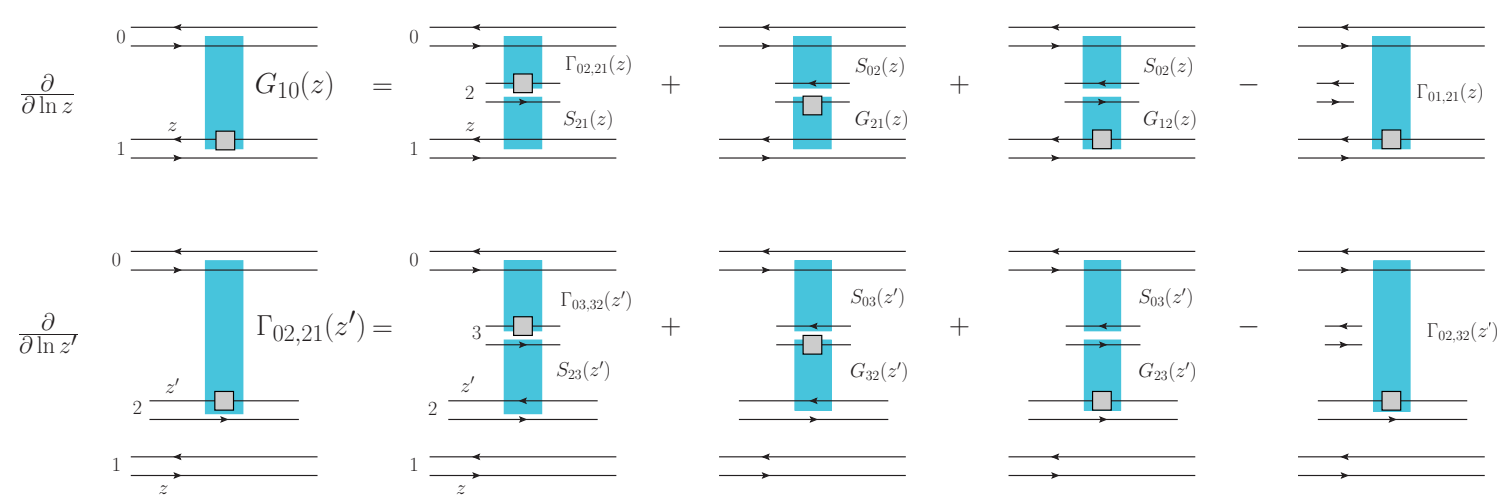

Figure 2: Large- $N_{c}$ helicity evolution for the polarized dipole amplitude $G$ and the neighbor dipole amplitude $\Gamma$. For pictorial simplicity we do not show the contributions of the initial condition terms. Double lines denote gluons at large $N_{c}$. Only one of the virtual diagrams is shown (last diagram in each line): virtual corrections to the right of the shock wave are implied, but not shown explicitly.

The evolution equation for $G_{10}(z)$ is illustrated in the top line of Fig. 2. Note that the large- $N_{c}$ limit is gluon-dominated: hence the dipole 10 is made out of quark and anti-quark lines of the 
$\operatorname{large}-N_{c}$ gluon. The equation reads $\left(x_{i j}=\left|\underline{x}_{i}-\underline{x}_{j}\right|, \rho^{\prime 2}=1 /\left(z^{\prime} s\right)\right)$

$$
\begin{aligned}
G_{10}(z)=G_{10}^{(0)}(z)+\frac{\alpha_{s} N_{c}}{2 \pi} \int_{z_{i}}^{z} \frac{d z^{\prime}}{z^{\prime}} \int_{\rho^{\prime 2}}^{x_{10}^{2}} \frac{d x_{21}^{2}}{x_{21}^{2}} & {\left[2 \Gamma_{02,21}\left(z^{\prime}\right) S_{21}\left(z^{\prime}\right)+2 G_{21}\left(z^{\prime}\right) S_{02}\left(z^{\prime}\right)\right.} \\
& \left.+G_{12}\left(z^{\prime}\right) S_{02}\left(z^{\prime}\right)-\Gamma_{01,21}\left(z^{\prime}\right)\right]
\end{aligned}
$$

where $\Gamma_{02,21}\left(z^{\prime}\right)$ is the new object (as compared to the unpolarized evolution), characteristic of helicity evolution. $\Gamma_{02,21}\left(z^{\prime}\right)$ is the "neighbor dipole" amplitude. Its evolution is described in the bottom line of Fig. 2. As shown in the figure, the "neighbor" dipole evolution continues in dipole 02, but the information about the dipole 21 comes in through the transverse size integration limit. (This is in contrast to unpolarized evolution, where the evolution in, say, dipole 02 does not depend on the size of the dipole 21 or on anything else outside the dipole 02.) The evolution for the neighbor dipole amplitude reads $\left(\rho^{\prime \prime 2}=1 /\left(z^{\prime \prime} s\right)\right)$

$$
\begin{aligned}
\Gamma_{02,21}\left(z^{\prime}\right)=\Gamma_{02,21}^{(0)}\left(z^{\prime}\right)+\frac{\alpha_{s} N_{c}}{2 \pi} \int_{z_{i}}^{z^{\prime}} \frac{d z^{\prime \prime}}{z^{\prime \prime}} & \int_{\rho^{\prime \prime 2}}^{\min \left\{x_{02}^{2}, x_{21}^{2} z^{\prime} / z^{\prime \prime}\right\}} \frac{d x_{32}^{2}}{x_{32}^{2}}\left[2 \Gamma_{03,32}\left(z^{\prime \prime}\right) S_{23}\left(z^{\prime \prime}\right)\right. \\
& \left.+2 G_{32}\left(z^{\prime \prime}\right) S_{03}\left(z^{\prime \prime}\right)+G_{23}\left(z^{\prime \prime}\right) S_{03}\left(z^{\prime \prime}\right)-\Gamma_{02,32}\left(z^{\prime \prime}\right)\right] .
\end{aligned}
$$

Eqs. (3.3) and (3.4), when augmented by the BK evolution for $S$, present a closed system of equations. The initial conditions $G^{(0)}$ and $\Gamma^{(0)}$ are given by the Born-level interactions, enhanced by multiple rescatterings which bring in saturation effects.

In the strict DLA limit we can simplify Eqs. (3.3) and (3.4) by putting $S=1$ and assuming that $G_{21}=G_{12}$. We obtain

$$
\begin{gathered}
G_{01}(z)=G_{01}^{(0)}(z)+\frac{\alpha_{s} N_{c}}{2 \pi} \int_{z_{i}}^{z} \frac{d z^{\prime}}{z^{\prime}} \int_{\rho^{\prime 2}}^{x_{10}^{2}} \frac{d x_{21}^{2}}{x_{21}^{2}}\left[\Gamma_{02,21}\left(z^{\prime}\right)+3 G_{21}\left(z^{\prime}\right)\right], \\
\Gamma_{02,21}\left(z^{\prime}\right)=\Gamma_{02,21}^{(0)}\left(z^{\prime}\right)+\frac{\alpha_{s} N_{c}}{2 \pi} \int_{z_{i}}^{z^{\prime}} \frac{d z^{\prime \prime}}{z^{\prime \prime}} \int_{\rho^{\prime \prime 2}}^{\min \left\{x_{02}^{2}, x_{21}^{2} z^{\prime} / z^{\prime \prime}\right\}} \frac{d x_{32}^{2}}{x_{32}^{2}}\left[\Gamma_{03,32}\left(z^{\prime \prime}\right)+3 G_{23}\left(z^{\prime \prime}\right)\right] .
\end{gathered}
$$

These equations are solved numerically in [2]. Interestingly, the resulting intercept for small- $x$ asymptotics of the flavor-singlet helicity observables is different from the one previously derived in [6] using the IR renormalization group approach. In [3] we identify DLA diagram contributions not included by the authors of [6] in their treatment of the problem.

\section{Large- $N_{c} \& N_{f}$ Limit}

Helicity evolution equations also close in the large- $N_{c} \& N_{f}$ limit. To write down these new evolution equations we need to define a couple of new objects. In addition to $G_{10}(z)$ defined in Eq. (2.2a) above, which is made out of quark and anti-quark lines of gluons (with $x_{1}$ line polarized), let us define

$$
A_{10}(z)=\frac{1}{2 N_{c}}\left\langle\left\langle\operatorname{tr}\left[V_{\underline{0}} V_{\underline{1}}^{p o l \dagger}\right]\right\rangle\right\rangle(z)+\frac{1}{2 N_{c}}\left\langle\left\langle\operatorname{tr}\left[V_{\underline{1}}^{p o l} V_{\underline{0}}^{\dagger}\right]\right\rangle\right\rangle(z)
$$


with $x_{1}$ being a true quark or anti-quark polarized line and $x_{0}$ being the (anti-)quark line of the gluon, and

$$
Q_{10}(z)=\frac{1}{2 N_{c}}\left\langle\left\langle\operatorname{tr}\left[V_{\underline{0}} V_{\underline{1}}^{p o l} \dagger\right]\right\rangle\right\rangle(z)+\frac{1}{2 N_{c}}\left\langle\left\langle\operatorname{tr}\left[V_{\underline{1}}^{p o l} V_{\underline{0}}^{\dagger}\right]\right\rangle\right\rangle(z)
$$

with both $x_{0}$ and $x_{1}$ being true quark and anti-quark lines and $x_{1}$ polarized.
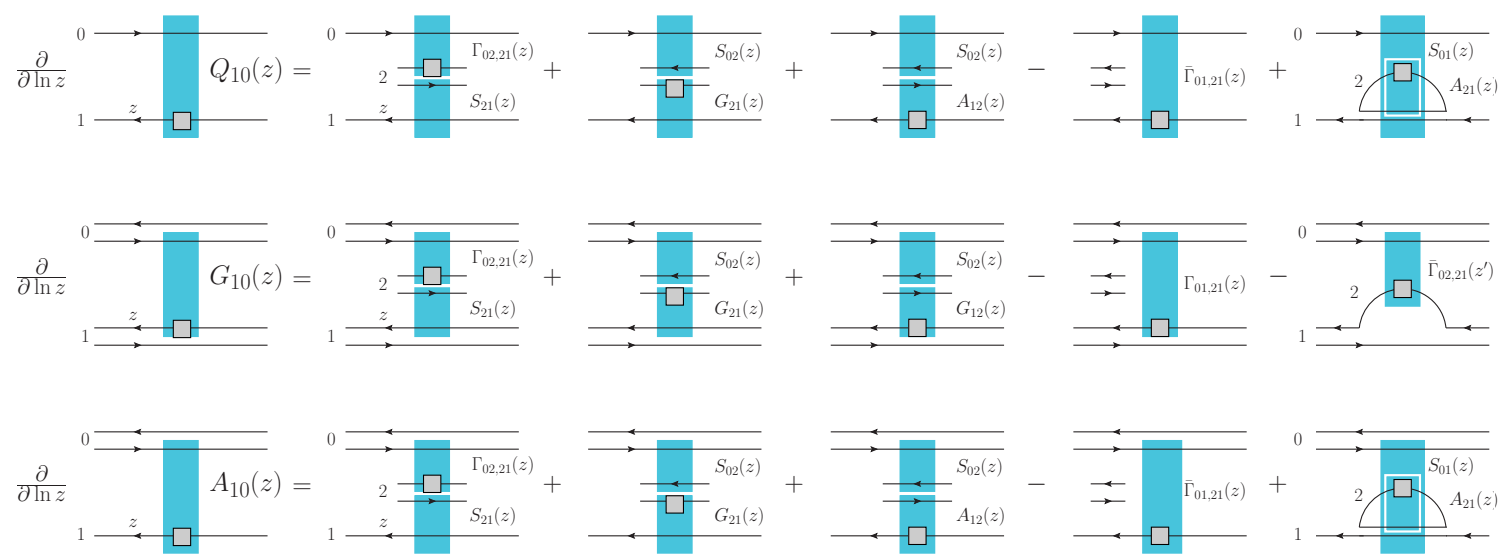

Figure 3: Large- $N_{c} \& N_{f}$ helicity evolution for the polarized dipole amplitudes $Q, G$ and $A$.

Large- $N_{c} \& N_{f}$ evolution equations for $Q, G$ and $A$ are illustrated diagrammatically in Fig. 3, where again we do not show the initial condition terms for simplicity. The equation for $Q$ is

$$
\begin{aligned}
Q_{10}(z)= & Q_{10}^{(0)}(z)+\frac{\alpha_{s} N_{c}}{2 \pi^{2}} \int_{z_{i}}^{z} \frac{d z^{\prime}}{z^{\prime}} \int_{\rho^{\prime 2}} \frac{d^{2} x_{2}}{x_{21}^{2}} \theta\left(x_{10}-x_{21}\right) \\
& \times\left[S_{21}\left(z^{\prime}\right) \Gamma_{02,21}\left(z^{\prime}\right)+S_{02}\left(z^{\prime}\right) G_{21}\left(z^{\prime}\right)+S_{02}\left(z^{\prime}\right) A_{12}\left(z^{\prime}\right)-\bar{\Gamma}_{01,21}\left(z^{\prime}\right)\right] \\
& +\frac{\alpha_{s} N_{c}}{4 \pi^{2}} \int_{z_{i}}^{z} \frac{d z^{\prime}}{z^{\prime}} \int_{\rho^{\prime 2}} \frac{d^{2} x_{2}}{x_{21}^{2}} \theta\left(x_{10}^{2} z-x_{21}^{2} z^{\prime}\right) S_{01}\left(z^{\prime}\right) A_{21}\left(z^{\prime}\right) .
\end{aligned}
$$

Equation (4.3) is illustrated diagrammatically in the first line of Fig. 3. The equation for $G$ is now

$$
\begin{aligned}
& G_{10}(z)=G_{10}^{(0)}(z)+\frac{\alpha_{s} N_{c}}{2 \pi^{2}} \int_{z_{i}}^{z} \frac{d z^{\prime}}{z^{\prime}} \int_{\rho^{\prime 2}} \frac{d^{2} x_{2}}{x_{21}^{2}} \theta\left(x_{10}-x_{21}\right) \\
& \times\left[2 S_{21}\left(z^{\prime}\right) \Gamma_{02,21}\left(z^{\prime}\right)+2 S_{02}\left(z^{\prime}\right) G_{21}\left(z^{\prime}\right)+S_{02}\left(z^{\prime}\right) G_{12}\left(z^{\prime}\right)-\Gamma_{01,21}\left(z^{\prime}\right)\right] \\
& -\frac{\alpha_{s} N_{f}}{4 \pi^{2}} \int_{z_{i}}^{z} \frac{d z^{\prime}}{z^{\prime}} \int_{\rho^{\prime 2}} \frac{d^{2} x_{2}}{x_{21}^{2}} \theta\left(x_{10}^{2} z-x_{21}^{2} z^{\prime}\right) \bar{\Gamma}_{02,21}\left(z^{\prime}\right) .
\end{aligned}
$$

Note a new object, $\bar{\Gamma}_{02,21}$, which is the neighbor dipole amplitude with line 2 being an actual polarized quark (or anti-quark), and, unlike in $\Gamma_{02,21}$, not a quark (or anti-quark) line of a large- $N_{c}$ gluon. Equation (4.4) is illustrated diagrammatically in the second line of Fig. 3. 
Finally, the evolution for $A_{01}(z)$ reads

$$
\begin{aligned}
& A_{10}(z)=A_{10}^{(0)}(z)+\frac{\alpha_{s} N_{c}}{2 \pi^{2}} \int_{z_{i}}^{z} \frac{d z^{\prime}}{z^{\prime}} \int_{\rho^{\prime 2}} \frac{d^{2} x_{2}}{x_{21}^{2}} \theta\left(x_{10}-x_{21}\right) \\
& \times\left[S_{21}\left(z^{\prime}\right) \Gamma_{02,21}\left(z^{\prime}\right)+S_{02}\left(z^{\prime}\right) G_{21}\left(z^{\prime}\right)+S_{02}\left(z^{\prime}\right) A_{12}\left(z^{\prime}\right)-\bar{\Gamma}_{01,21}\left(z^{\prime}\right)\right] \\
& +\frac{\alpha_{s} N_{c}}{4 \pi^{2}} \int_{z_{i}}^{z} \frac{d z^{\prime}}{z^{\prime}} \int_{\rho^{\prime 2}} \frac{d^{2} x_{2}}{x_{21}^{2}} \theta\left(x_{10}^{2} z-x_{21}^{2} z^{\prime}\right) S_{01}\left(z^{\prime}\right) A_{21}\left(z^{\prime}\right) .
\end{aligned}
$$

It is depicted in the last line of Fig. 3.

Note that Eq. (3.4) for the neighbor dipole amplitude also has to be modified yielding

$$
\begin{aligned}
& \Gamma_{02,21}\left(z^{\prime}\right)=\Gamma_{02,21}^{(0)}\left(z^{\prime}\right)+\frac{\alpha_{s} N_{c}}{2 \pi} \int_{z_{i}}^{z^{\prime}} \frac{d z^{\prime \prime}}{z^{\prime \prime}} \int_{\rho^{\prime \prime 2}}^{\min \left\{x_{02}^{2}, x_{21}^{2} z^{\prime} / z^{\prime \prime}\right\}} \frac{d x_{32}^{2}}{x_{32}^{2}} \\
& \times\left[2 \Gamma_{03,32}\left(z^{\prime \prime}\right) S_{23}\left(z^{\prime \prime}\right)+2 G_{32}\left(z^{\prime \prime}\right) S_{03}\left(z^{\prime \prime}\right)+G_{23}\left(z^{\prime \prime}\right) S_{03}\left(z^{\prime \prime}\right)-\Gamma_{02,32}\left(z^{\prime \prime}\right)\right] \\
& -\frac{\alpha_{S} N_{f}}{4 \pi} \int_{z_{i}}^{z^{\prime}} \frac{d z^{\prime \prime}}{z^{\prime \prime}} \int_{\rho^{\prime \prime 2}}^{x_{21}^{2} z^{\prime} / z^{\prime \prime}} \frac{d x_{32}^{2}}{x_{32}^{2}} \bar{\Gamma}_{03,32}\left(z^{\prime}\right) .
\end{aligned}
$$

We also need an equation for $\bar{\Gamma}$ :

$$
\begin{aligned}
& \bar{\Gamma}_{02,21}\left(z^{\prime}\right)=\bar{\Gamma}_{02,21}^{(0)}\left(z^{\prime}\right)+\frac{\alpha_{s} N_{c}}{2 \pi} \int_{z_{i}}^{z^{\prime}} \frac{d z^{\prime \prime}}{z^{\prime \prime}} \int_{\rho^{\prime \prime 2}}^{\min \left\{x_{02}^{2}, x_{21}^{2} z^{\prime} / z^{\prime \prime}\right\}} \frac{d x_{32}^{2}}{x_{32}^{2}} \\
& \times\left[\Gamma_{03,32}\left(z^{\prime \prime}\right) S_{23}\left(z^{\prime \prime}\right)+G_{32}\left(z^{\prime \prime}\right) S_{03}\left(z^{\prime \prime}\right)+A_{23}\left(z^{\prime \prime}\right) S_{03}\left(z^{\prime \prime}\right)-\bar{\Gamma}_{02,32}\left(z^{\prime \prime}\right)\right] \\
& +\frac{\alpha_{S} N_{c}}{4 \pi} \int_{z_{i}}^{z^{\prime}} \frac{d z^{\prime \prime}}{z^{\prime \prime}} \int_{\rho^{\prime \prime 2}}^{x_{21}^{2} z^{\prime} / z^{\prime \prime}} \frac{d x_{32}^{2}}{x_{32}^{2}} S_{02}\left(z^{\prime}\right) A_{32}\left(z^{\prime}\right) .
\end{aligned}
$$

Both of these equations are diagrammatically illustrated in Fig. 4.

Equations (4.3), (4.4), (4.5), (4.6), and (4.7) are the large- $N_{c} \& N_{f}$ helicity evolution equations which are DLA in polarization-dependent terms, but also include LLA saturation corrections through the $S$-matrices.

In the pure DLA limit we linearize all these equations by putting $S=1$ in them (we again 

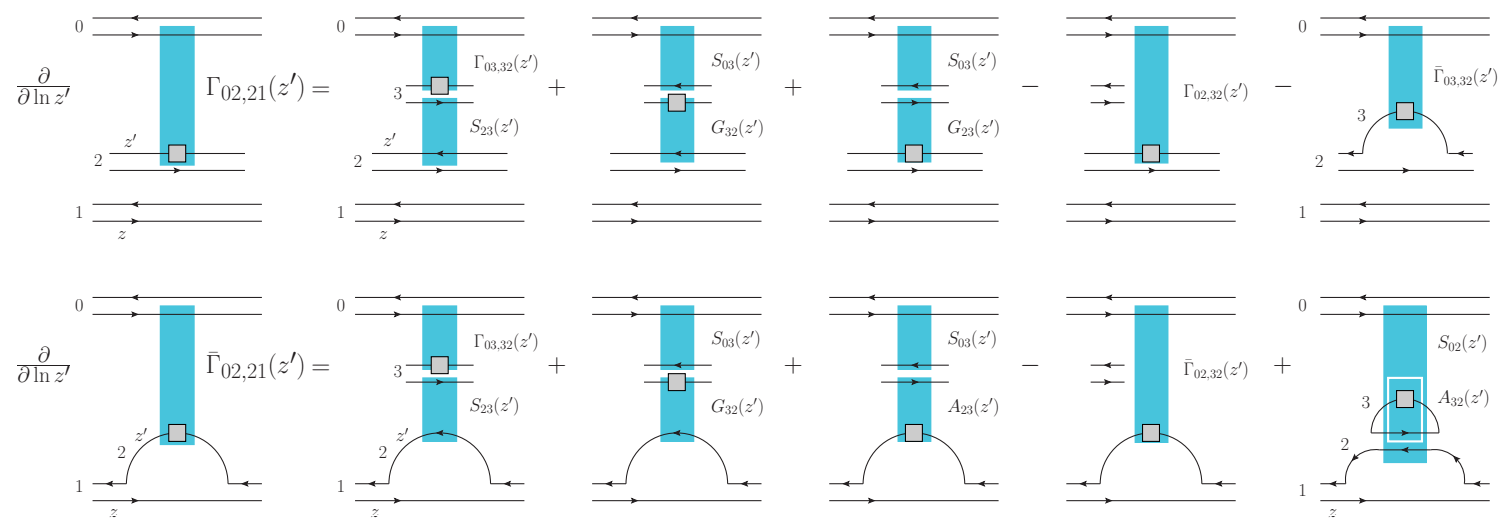

Figure 4: Large- $N_{c} \& N_{f}$ helicity evolution for the polarized neighbor dipole amplitudes $\Gamma$ and $\bar{\Gamma}$.

assume that $G_{01}=G_{10}$, which is true for a large, longitudinally polarized target):

$$
\begin{aligned}
Q_{01}(z)= & Q_{01}^{(0)}(z)+\frac{\alpha_{s} N_{c}}{2 \pi^{2}} \int_{z_{i}}^{z} \frac{d z^{\prime}}{z^{\prime}} \int_{\rho^{\prime 2}} \frac{d^{2} x_{2}}{x_{21}^{2}} \theta\left(x_{10}-x_{21}\right)\left[G_{12}\left(z^{\prime}\right)+\Gamma_{02,21}\left(z^{\prime}\right)+A_{21}\left(z^{\prime}\right)-\bar{\Gamma}_{01,21}\left(z^{\prime}\right)\right] \\
& +\frac{\alpha_{s} N_{c}}{4 \pi^{2}} \int_{z_{i}}^{z} \frac{d z^{\prime}}{z^{\prime}} \int_{\rho^{\prime 2}} \frac{d^{2} x_{2}}{x_{21}^{2}} \theta\left(x_{10}^{2} z-x_{21}^{2} z^{\prime}\right) A_{21}\left(z^{\prime}\right), \\
G_{10}(z)= & G_{10}^{(0)}(z)+\frac{\alpha_{s} N_{c}}{2 \pi^{2}} \int_{z_{i}}^{z} \frac{d z^{\prime}}{z^{\prime}} \int_{\rho^{\prime 2}} \frac{d^{2} x_{2}}{x_{21}^{2}} \theta\left(x_{10}-x_{21}\right)\left[\Gamma_{02,21}\left(z^{\prime}\right)+3 G_{12}\left(z^{\prime}\right)\right] \\
& -\frac{\alpha_{s} N_{f}}{4 \pi^{2}} \int_{z_{i}}^{z} \frac{d z^{\prime}}{z^{\prime}} \int_{\rho^{\prime 2}} \frac{d^{2} x_{2}}{x_{21}^{2}} \theta\left(x_{10}^{2} z-x_{21}^{2} z^{\prime}\right) \bar{\Gamma}_{02,21}\left(z^{\prime}\right), \\
A_{01}(z)= & A_{01}^{(0)}(z)+\frac{\alpha_{s} N_{c}}{2 \pi^{2}} \int_{z_{i}}^{z} \frac{d z^{\prime}}{z^{\prime}} \int_{\rho^{\prime 2}} \frac{d^{2} x_{2}}{x_{21}^{2}} \theta\left(x_{10}-x_{21}\right)\left[G_{12}\left(z^{\prime}\right)+\Gamma_{02,21}\left(z^{\prime}\right)+A_{21}\left(z^{\prime}\right)-\bar{\Gamma}_{01,21}\left(z^{\prime}\right)\right] \\
& +\frac{\alpha_{s} N_{c}}{4 \pi^{2}} \int_{z_{i}}^{z} \frac{d z^{\prime}}{z^{\prime}} \int_{\rho^{\prime 2}} \frac{d^{2} x_{2}}{x_{21}^{2}} \theta\left(x_{10}^{2} z-x_{21}^{2} z^{\prime}\right) A_{12}\left(z^{\prime}\right) .
\end{aligned}
$$


The linearized equations for $\Gamma$ and $\bar{\Gamma}$ in the large- $N_{c} \& N_{f}$ limit become

$$
\begin{aligned}
& \Gamma_{02,21}\left(z^{\prime}\right)=\Gamma_{02,21}^{(0)}\left(z^{\prime}\right)+\frac{\alpha_{s} N_{c}}{2 \pi} \int_{z_{i}}^{z^{\prime}} \frac{d z^{\prime \prime}}{z^{\prime \prime}} \int_{\rho^{\prime \prime 2}}^{\min \left\{x_{02}^{2}, x_{21}^{2} z^{\prime} / z^{\prime \prime}\right\}} \frac{d x_{32}^{2}}{x_{32}^{2}}\left[\Gamma_{03,32}\left(z^{\prime \prime}\right)+3 G_{23}\left(z^{\prime \prime}\right)\right] \\
& -\frac{\alpha_{S} N_{f}}{4 \pi} \int_{z_{i}}^{z^{\prime}} \frac{d z^{\prime \prime}}{z^{\prime \prime}} \int_{\rho^{\prime \prime 2}}^{x_{21}^{2} z^{\prime} / z^{\prime \prime}} \frac{d x_{32}^{2}}{x_{32}^{2}} \bar{\Gamma}_{03,32}\left(z^{\prime}\right) \\
& \bar{\Gamma}_{02,21}\left(z^{\prime}\right)=\bar{\Gamma}_{02,21}^{(0)}\left(z^{\prime}\right)+\frac{\alpha_{s} N_{c}}{2 \pi} \int_{z_{i}}^{z^{\prime}} \frac{d z^{\prime \prime}}{z^{\prime \prime}} \int_{\rho^{\prime \prime 2}}^{\min \left\{x_{02}^{2}, x_{21}^{2} z^{\prime} / z^{\prime \prime}\right\}} \frac{d x_{32}^{2}}{x_{32}^{2}}\left[\Gamma_{03,32}\left(z^{\prime \prime}\right)+G_{23}\left(z^{\prime \prime}\right)+A_{23}\left(z^{\prime \prime}\right)\right. \\
& \left.-\bar{\Gamma}_{02,32}\left(z^{\prime \prime}\right)\right]+\frac{\alpha_{s} N_{c}}{4 \pi} \int_{z_{i}}^{z^{\prime}} \frac{d z^{\prime \prime}}{z^{\prime \prime}} \int_{\rho^{\prime \prime 2}}^{x_{21}^{2} z^{\prime} / z^{\prime \prime}} \frac{d x_{32}^{2}}{x_{32}^{2}} A_{32}\left(z^{\prime}\right)
\end{aligned}
$$

Note that in the large- $N_{c} \& N_{f}$ limit Eqs. (2.1), (2.7) and (2.8) should contain $Q_{10}(z)$ instead of $G_{10}(z)$.

Clearly in the large- $N_{c} /$ fixed- $N_{f}$ limit the linearized equations for $G_{01}(z)$ and $\Gamma_{02,21}\left(z^{\prime}\right)$ become a closed system of equations (3.5) again, as employed in the previous Subsection. Since our final observable, quark helicity TMD or hPDF, is related to $Q$, for the large- $N_{c}$ limit to be relevant, $G$ should dominate (or at least be comparable to) $A$.

The linearized equations (4.8) and (4.9), when solved, should yield the helicity evolution intercept in the large- $N_{c} \& N_{f}$ limit. Solution of Eqs. (4.8) and (4.9) is left for the future (probably numerical) work.

\section{Summary}

We have derived small- $x$ evolution equations for the polarized dipole amplitude. The equations are given above, and close in the large- $N_{c}$ and large- $N_{c} \& N_{f}$ limits. The solution of these equations provides theoretical input on the perturbative value of the small- $x$ intercept for the quark helicity TMD and PDF, and for the $g_{1}$ structure function.

\section{Acknowledgments}

This material is based upon work supported by the U.S. Department of Energy, Office of Science, Office of Nuclear Physics under Award Number DE-SC0004286 (YK) and within the framework of the TMD Topical Collaboration (DP) and DOE Contract No. DE-SC0012704 (MS). DP also received support from the RIKEN BNL Research Center. MS received additional support from an EIC program development fund from BNL and from the U.S. Department of Energy, Office of Science under the DOE Early Career Program. 


\section{References}

[1] Y. V. Kovchegov, D. Pitonyak and M. D. Sievert, JHEP 1601, 072 (2016) doi:10.1007/JHEP01(2016)072 [arXiv:1511.06737 [hep-ph]].

[2] Y. V. Kovchegov, D. Pitonyak and M. D. Sievert, arXiv:1610.06188 [hep-ph].

[3] Y. V. Kovchegov, D. Pitonyak and M. D. Sievert, arXiv:1610.06197 [hep-ph].

[4] Y. V. Kovchegov and M. D. Sievert, Nucl. Phys. B 903, 164 (2016) doi:10.1016/j.nuclphysb.2015.12.008 [arXiv:1505.01176 [hep-ph]].

[5] K. Itakura, Y. V. Kovchegov, L. McLerran and D. Teaney, Nucl. Phys. A 730, 160 (2004) doi:10.1016/j.nuclphysa.2003.10.016 [hep-ph/0305332].

[6] J. Bartels, B. Ermolaev, and M. Ryskin, Flavor singlet contribution to the structure function $G(1)$ at small x, Z.Phys. C72 (1996) 627-635, [hep-ph/ 9603204 ]. 